\title{
Astrometry of Southern Pulsars
}

\author{
David Legge \\ School of Physics, University of Tasmania, Hobart TAS 7001, Australia
}

\begin{abstract}
A preliminary determination of the proper motion and parallax of the Vela pulsar was made using phase-referencing VLBI. The measured proper motion in right ascension and declination are respectively, $\mu_{\alpha^{-}}=-49.8 \pm 0.2$ mas yr $^{-1}$ and $\mu_{\delta}=30.5 \pm 0.1$ mas yr $^{-1}$. The parallax of Vela was calculated to be $\pi_{\text {Vela }}=3.16 \pm 0.33$ mas, which corresponds to a distance of $D_{\text {Vela }}=316_{-29}^{+37} \mathrm{pc}$.
\end{abstract}

\section{Observations \& Data Reduction}

Multiple-epoch observations of the Vela pulsar were conducted using elements of the Australian and South African Long Baseline Array (LBA), as shown in Table 1. Phase-referencing observations were performed every $2.5 \mathrm{~min}$ between Vela and extragalactic source Vela-G. Vela- $G$ was phase referenced by Vela at $2.3 \mathrm{GHz}$, and Vela was phase referenced by Vela- $\mathrm{G}$ at $8.4 \mathrm{GHz}$. Single frequency Mark-III data were correlated at Penticton, Canada, while dual frequency S2 data were correlated at ATNF's LBA correlator in Sydney. The LBA correlator can bin products in up to 32 period-synchronous time bins. Vela's pulse width at $2.3 \mathrm{GHz}$ is 2.5 bins, and at $8.4 \mathrm{GHz}$ it is $\approx 1$ bin. The $\mathrm{S} 2$ data was processed using an ATNF customized version of NRAO's Astronomical Image Processing Software (AIPS). Using standard VLBI techniques, the data for the phase reference source for each epoch were delay and phase-calibrated, and solutions were applied to the target source. The relative offset between the target source and phase-reference source was measured for each epoch. This allows the position of the target source to be measured over time.

\section{Results \& Discussion}

The motion of the Vela pulsar has a proper motion and parallax component. The parallax is a time-variable projection of the Earth's orbit onto the sky, tracing a sinusoid in RA and Declination through time. The proper motion and parallax contributions were determined from an unwieghted, least squares minimization. The caculated proper motions in right ascension and declination are $\mu_{\alpha} \equiv \dot{\alpha} \cos \delta=-49.8 \pm 0.2 \mathrm{mas} \mathrm{yr}^{-1}$ and $\mu_{\delta}=30.5 \pm 0.1 \mathrm{mas} \mathrm{yr}^{-1}$, respectively. The parallax of Vela: $\pi_{\text {Vela }}=3.16 \pm 0.33$ mas, which corresponds to a distance of $D_{\text {Vela }}=316_{-29}^{+37} \mathrm{pc}$. The tangential velocity of the Vela pulsar is $v_{\mathrm{T}}=104 \mathrm{~km} \mathrm{~s}^{-1}$

The proper motion agrees reasonably well with that of Bailes et al. (1989). The distance of $316 \mathrm{pc}$ is much less than early estimates of $400-600 \mathrm{pc}$ found by 
Table 1. Observational parameters

\begin{tabular}{lllcl}
\hline \hline Expt & Epoch & Rec. mode & $\begin{array}{c}\text { Freq. } \\
(\mathrm{GHz})\end{array}$ & Telescopes \\
\hline V041A & 25 Apr 1993 & Mk-III & 2.3 & $\begin{array}{l}\text { DSS43,Parkes,Hobart, } \\
\text { Hartbeesthoek }\end{array}$ \\
V041C & 18 Jun 1994 & Mk-III & 2.3 & $\begin{array}{l}\text { DSS43, DSS45, Parkes, } \\
\text { Hobart,Hartbeesthoek }\end{array}$ \\
& & & & DSS43, Hobart \\
V041G & 22 Apr 1995 & Mk-III & $2.3 / 8.4$ & D \\
V041L & 17 Jul 1996 & Mk-III/S2 & $2.3 / 8.4$ & DSS43,Hobart \\
V041M & 23 Nov 1996 & Mk-III/S2 & $2.3 / 8.4$ & DSS43,Hobart \\
V041O & 26 May 1997 & S2 & $2.3 / 8.4$ & DSS43,Hobart \\
V041P & 26 Mar 1998 & S2 & $2.3 / 8.4$ & DSS43, Hobart \\
V041S & 17 Feb 1999 & S2 & $2.3 / 8.4$ & DSS43,Hobart \\
\hline
\end{tabular}

DSS43 \& DSS45 are at Tidbinbilla

Milne (1968), Prentice \& ter Haar (1969), and Davies (1969). Recent estimates using a variety of techniques have had average values in the range $230-290 \mathrm{pc}$ (Ogelman et al. 1989; Oberlack et al. 1994; Jenkins \& Wallerstein 1995; Cha, Sembach, \& Danks 1999), which are consistant with the results presented here.

Acknowledgments. I wish to thank M. Bailes (Swinburne Univ.), M. Costa (Univ. of Tas.), R. Dodson (Univ. of Tas.), P. McCulloch (Univ. of Tas.), D. Moffett (Univ. of Tas.), J. Reynolds (ATNF), and the staff of the ATNF for assistance with observations, data correlation and processing.

\section{References}

Australian Pulsar Timing Archive ${ }^{1}$

Bailes, M., Manchester, R. N., Kesteven, M. J., Norris, R. P., \& Reynolds, J. E. 1989, ApJ, 343, L53

Cha, A. N., Sembach, K. R., \& Danks, A. C. 1999, ApJ, 515, L25

Davies, R. D. 1969, Nature, 223, 355

Jenkins, E., \& Wallerstein, G. 1995, ApJ 440, 227

Milne, D. K. 1968, Australian J. Phys., 21, 201

Oberlack, U., Diehl, R., Montmerle, T., Prantzos, N., \& von Ballmoos, P. 1994, ApJS, 92, 433

Ögelman, H., Koch-Miramond, L., \& Auriére, M. 1989, ApJ 342, L83

Prentice A., \& ter Haar, D. 1969, Nature, 222, 964

${ }^{1}$ http://wwwatnf.atnf.csiro.au/Research/pulsar/psr/archive/ 